results may be interpreted as lending support to the suggestion put forward by Stekol ${ }^{5}$, which has been substantiated by the results of work by Bloch and Rittenberg 7 , that the biological processes for acetylating aliphatic and aromatic amines are separate processes.

A fuller report of this investigation is in preparation for publication.

Physiology Department, Medical School,

University of Birmingham. August 27.

' smith, J. N., and Williams, R. T., Biochem. J., 42, 538 (1948).

' Jafié, M., and Hilbert, P., Hoppe-Seyl. Z., 12, 295 (1888).

${ }^{3}$ Marshall, E. K., Emerson, K., and Cutting, W. C., Science, 85, 202 (1937).

4 Stokinger, H. E., Proc. Soc. Exp. Biol., N.Y., 40, 61 (1939).

Stekol, J. A., J. Biol. Chem., 124. 129 (1938).

- Krebs, H. A., Sykes, W. O., and Bartley, W. C., Biochem. J., 41, 622 (1947).

'Bloch, K., and Rittenberg, D., J. Biol. Chem., 1:9, 45 (1945); Fed. Proc., 5, 122 (1946)

\section{Role of Hydroxylamine in Biological Fixation of Nitrogen}

THE position of hydroxylamine as an intermediate in the nitrogen fixation process has become increasingly doubtful as a result of recent studies ${ }^{1,2}$. No direct attempts have been made, however, to find the effect of hydroxylamine on nitrogen fixation. An application of the isotope tracer method to this problem is being made in these laboratories, using nitrogen containing 30 per cent nitrogen-15. A large 18-hourold culture of Azotobacter vinelandii in a nitrogen-free mannitol medium was aseptically subdivided into equal $100-\mathrm{ml}$. portions, and $1 \mathrm{ml}$. of suitable dilutions of buffered hydroxylamine added to give the concentrations required for inhibition measurements. The cultures were then aerated in parallel with an atmosphere containing nitrogen (partial pressure, 0.25 atm.), and oxygen (partial pressure, $0.2 \mathrm{~atm}$.)--the nitrogen being 'heavy'-in a thermostat at $29^{\circ} \mathrm{C}$. After a four-hour period of aeration, the cells were collected, and nitrogen samples prepared for isotope analysis. The following analyses were obtained :

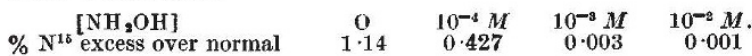

This represents 63 per cent inhibition of nitrogen uptake at $10^{-4} M$, and virtually 100 per cent inhibition at $10^{-3} M$ and higher. Hydroxylamine is well known to be a powerful respiratory inhibitor ${ }^{3}$, and since nitrogen fixation is an endothermic process, it should be inhibited also'. If hydroxylamine is an intermediate in the fixation process, it can be expected to replace molecular nitrogen in the metabolism of the bacteria, and this effect will, therefore, be reflected in greater inhibition of nitrogen fixation than respiration, for a given concentration of hydroxylamine. A provisional comparison may be made with the results of respiration experiments of other workers. Wilson and Wilson ${ }^{3}$ report 90-100 per cent inhibition at $10^{-3} \mathrm{M}$, and 90 per cent at $10^{-4} \mathrm{M}$, with Azotobacter vinelandii in a succinate medium. Endres ${ }^{4}$ reports approximately 60 per cent inhibition at $10^{-4} M$, and 90 per cent at $10^{-3}$, with Azotobacter chroococcum in a glucose medium. The inhibition of nitrogen fixation, therefore, does not appear to be greater than the inhibition of respiration, although a final comparison cannot be made until experiments designed to permit this, and proceeding here, have been completed with the strain of Azotobacter available.

Parallel experiments are being made to find the effect of hydroxylamine on the growth of Azotobacter on gaseous nitrogen. The organisms were cultured in various concentrations of hydroxylamine, and growth measured by direct counting. Growth was supported indefinitely in a solution of initial concentration $10^{-4}$ $M$, for one subculture only at $10^{-3} M$, and not at all at $10^{-2} M$. Long lag phases were observed, however, growth being apparently delayed until the concentration of hydroxylamine had decreased to less than $5 \times 10^{-5} M$ in the first subculture, which agrees with the observations of Novak and Wilson ${ }^{2}$. The lag increased from a few hours in the first subculture at $10^{-4} \mathrm{M}$, to three days at the sixth. Further experiments, which are being continued, indicate that the rate of removal of hydroxylamine from a culture medium is independent of the presence of the bacteria, even with six previous subcultures at $10^{-4} M$ (comparing with Lewis and Hinshelwood's observations ${ }^{5}$ ). Experiments here have shown that the levels of ammonia in culture solutions of Azotobacter supplied with hydroxylamine were higher than those not similarly supplied. This, together with the above observations on the rates of removal of hydroxylamine from such solution, suggests the liberation of nitrogenous materials from the cells in the presence of hydroxyl. amine, rather than the reduction of the latter, as postulated for the symbiotic system by Virtanen". The liberation of nitrogenous substances was confirmed by Kjeldahl analyses.

The results of these studies support those from the isotope-tracing experiment above in suggesting that hydroxylamine is unlikely to be one of the stable intermediates of nitrogen fixation by Azotobacter.

The isotope abundance measurements were made by one of us (E. R. S. W.) on a Nier-type, $60^{\circ}$ sector mass spectrometer, with electronic circuits described by Graham et al. ${ }^{7}$. We wish to express our indebted. ness to Dr. S. E. Jacolos for his continued interest and advice.

\section{B. A. Pethica \\ E. R. ROBERTS \\ E. R. S. WINTER}

Imperial College of Science and Technology, London, S.W.7. Aug. 31.

' Wilson, P. W., and Burris, R. H., Bact. Rev., 11, 41 (1947).

Novak, R., and Wilson, P. W., J. Bact., 55, 517 (1948).

${ }^{3}$ Wilson, J. B., and Wilson, P. W., J. Gen. Physiol., 26, 277 (1943) - Endres, G., Ann. Chem., 518. 109 (1935).

${ }^{5}$ Lewis, P. R., and Hinshelwood, C. N., J. Chem. Soc., 824 (1948)

- Virtanen, A. I., et al., Suomen Kemistilehti, 19. 83 (1946).

' Graham, R. L., Thode, H. G., and Harkness, A. I., J. Sci. Instr. $24,119(1947)$.

\section{Effect of $\mathrm{pH}$ on the Activity of some Respiratory Inhibitors}

Changes of $p H$ have been shown to influence the activity of certain respiratory inhibitors, for example, iodoacetate $^{1}$, azide ${ }^{2}$, fluoride ${ }^{3}$ and malonate ${ }^{4}$. We have been led to a detailed investigation of the effects of $p \mathrm{H}$ change on some of these inhibitors since they are all the salts of weak acids, and one of us (E. W. S.) has recently examined the influence of $p H$ on the fungistatic action of weak acids allied to phenol ${ }^{5}$.

The Warburg manometric technique was used to measure the rates of oxygen uptake by suspensions of 